Switchable selectivity within a series of boronate esters for dynamic covalent exchange in nonaqueous solvents

Stefan Borsley, Guillaume Poss, Rebecca L. Spicer, Eloïse Boudin and Euan R. Kay

EaStCHEM School of Chemistry, University of St Andrews, North Haugh, St Andrews, KY16 9ST, UK.

ek28@st-andrews.ac.uk 


\title{
Switchable selectivity within a series of boronate esters for dynamic covalent exchange in nonaqueous solvents
}

\author{
The reversible condensation-hydrolysis reactions of boronic acids have proven \\ to be a highly useful class of thermodynamically controlled dynamic covalent \\ process, enabling the construction of sugar sensors, stimuli-responsive materials, \\ and complex covalent architectures. Yet, the common diol or diphenol coupling \\ partners tend to produce relatively unstable condensation products, exhibit \\ oxidative sensitivity, or offer limited options for expanding structural diversity. \\ To address these drawbacks, we explore a series of coupling partners including \\ non-diol salicylate and salicylamide derivatives, in combination with two boronic \\ acids. In nonaqueous solvents, the condensation-hydrolysis equilibria are \\ sensitive to the nature and concentration of Lewis bases, with equilibrium \\ constants that can be tuned across at least five orders of magnitude. \\ Furthermore, differential responses to base concentration can be exploited to \\ create a switchable dynamic covalent system in which a boronic acid can be \\ cycled between expressing each of two condensation products with high fidelity, \\ in response to a simple chemical stimulus.
}

Keywords: dynamic covalent chemistry; adaptive systems; constitutional dynamic chemistry; salicylates; catechols; boronate esters; boronic acids; boronic esters

\section{Introduction}

The esters and anhydrides formed by boronic acids have received considerable attention as dynamic covalent functional groups.(1-2) They have been exploited, for example, to construct complex molecular architectures,(3) or extended crystalline organic frameworks;(4) to detect sugars,(5) or to actuate stimuli-responsive materials and drugdelivery devices.(6) The majority of these studies focus on aqueous systems, and particularly the reactions between boronic acids and aliphatic 1,2- or 1,3-dihydroxy motifs on saccharides or glycosylated biomolecules.(7) Although stabilized by chelate cooperativity, the resulting cyclic boronate esters tend to be labile, and display only 
moderate stabilities that are $\mathrm{pH}$ dependent.(7-8) Exquisite cooperativity effects in a handful of specific molecular architectures can lead to high stability,(3) while anhydrous aprotic solvents can maintain boronic ester stability, but typically then requiring harsh reaction conditions (e.g. solvothermal approaches) to achieve reversible covalent exchange. $(3,9)$

Considerable efforts have been devoted to increasing boronate ester stability through modification of the boron functional group,(10) or by introducing stabilizing intramolecular interactions.(11) Yet these systems are synthetically demanding and therefore remain relatively specialized. Despite significant early work on the reactions of boric acid, and sporadic reports spread over several decades since then, far less attention has been given to moving away from the common dihydroxy coupling partners. Furthermore, the challenges associated with understanding the multiple interconnected equilibria and system-dependent mechanistic details, have also encouraged a focus on phenomenology at the expense of detailed characterization at the molecular level, and most systematic studies have been carried out in aqueous systems. $(7-8,12)$ Attracted by the potential for orthogonality to common biomolecular functional groups and established bioorthogonal chemistries, recently Hall and coworkers have developed conformationally preorganized aliphatic diols that rapidly form stable boronate esters in water with an optimized boronic acid ligation partner,(13) while Anslyn and James have developed a three-component boronate ester coupling strategy using 2-formylphenylboronic acid to link aromatic-1,2-diols (catechols) with hydroxylamines.(14) Meanwhile, several groups have exploited boronate complexes with salicylhydroxamic acid derivatives for $\mathrm{pH}$-responsive gelation, bioseparation, drug delivery, and bioconjugation applications.(15) These advances suggest significant potential for discovering boronic acid coupling partners with superior characteristics for 
exploiting the reversibility of boronate ester formation in nonaqueous dynamic covalent applications.

It has long been recognized that catechols commonly exhibit higher boronate ester formation constants than their aliphatic analogues.(16) Despite their successful application for the construction of covalent organic architectures and frameworks, $(3-4)$ and stimuli-responsive polymers or soft nanomaterials, (6) these derivatives are challenging to adopt more generally, on account of their facile, irreversible aerobic oxidation to form quinones,(17) and a lack of synthetically amenable routes for derivatization. As part of our wider efforts to exploit dynamic covalent reactivity for surface-engineering of monolayer-stabilized nanoparticles, $(18)$ we have studied thermodynamically controlled boronate ester formation and exchange between catechols and nanoparticle-bound boronic acids, which we subsequently applied to direct reversible assembly and disassembly of covalently linked nanoparticle aggregates.(18b) The most reproducible results were achieved using relatively high concentrations of tertiary amine Lewis bases in organic solvents (e.g. $900 \mathrm{mM} \mathrm{N}$ methylmorpholine in methanol). Under these highly basic conditions, catechol oxidation is relatively rapid, while the structural scope of catechols that can be readily accessed for nanoparticle functionalization is limited. These issues have since led us to investigate alternative coupling partners for boronic acids, seeking higher boronate ester formation constants at lower concentrations of base, improved stability to aerobic oxidation, and structures which might be more amenable to functionalisation. We report here a comparative study of four coupling partners, their reversible covalent reactions with two model boronic acids (Scheme 1), and quantitative assessment of boronate ester formation constants at various concentrations of Lewis base. This investigation has allowed us to design a switchable dynamic covalent system whereby a boronic acid 
cycles between selecting each of two coupling partners in response to a simple chemical stimulus.

\section{Results and Discussion}

Boronic acids 1 and $\mathbf{2}$ (Scheme 1 and Chart S1) were designed as representative electron rich (1) and electron poor (2) small-molecule models of functionalized phenylboronic acids such as those we have previously incorporated in the monolayer of colloidal nanoparticles.(18b) As coupling partners, 4-fluorocatechol A provides a direct comparison with our nanoparticle-bound work, while the more acidic nitro-substituted analogue $\mathbf{B}$ probes the effect of catechol $\mathrm{p} K_{\mathrm{a}}$. Motivated by the drawbacks of catechol coupling partners (vide supra), and inspired by the recent reports using salicylhydroxamic acids in aqueous systems, (15) as well as earlier studies on esters of boric acid (also in water),(19) we decided to compare the catechols with salicylic acid and salicylamide scaffolds, $\mathbf{C}$ and $\mathbf{D}$ respectively. These derivatives were chosen for their resistance to aerobic oxidation, ease of preparation from readily available precursors, and routes to structural elaboration.
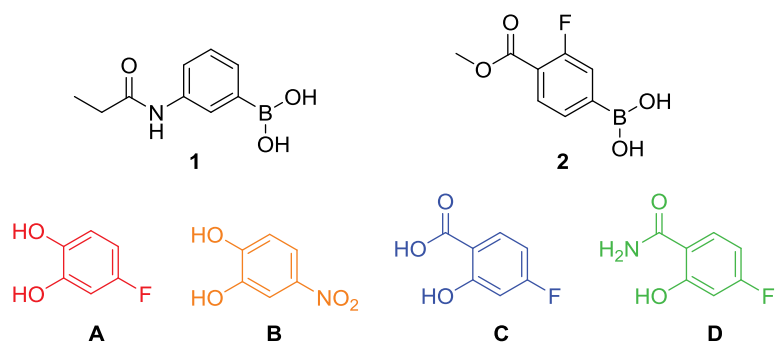

Scheme 1. Molecular structures of model electron rich (1) and electron poor (2) phenylboronic acids; catechol (A, B), salicylic acid (C) and salicylamide (D) coupling partners. See Chart S1 for the structures of all boronate ester complexes studied.

Boronate ester formation was investigated using ${ }^{19} \mathrm{~F}$ and ${ }^{1} \mathrm{H}$ NMR spectroscopy in $\mathrm{CD}_{3} \mathrm{OD}$ for a 1:1 stoichiometric ratio of boronic acid and coupling partner, each at ca. 
$5 \mathrm{mM}$. The complexes and their constituent components were observed to be in slow exchange on the NMR timescale, so that signals corresponding to each species could be independently identified (Figures 1 and S1-S7), and their concentrations determined by signal deconvolution relative to an internal standard. Electrospray mass spectrometry (Figures S21-S24) provided corroborating evidence that the complexes observed by NMR correspond to the boronate ester structures described in Chart S1, Figures 1 and 2.

On mixing boronic acid $\mathbf{1}$ with 4 -fluorocatechol $\mathbf{A}$, a very small $\operatorname{shift}(\Delta \delta<0.01$ ppm $)$ in the ${ }^{19} \mathrm{~F}$ NMR signal for the catechol component $(\delta=-124.4$ ppm, Figure 1a and S1) was observed, perhaps indicating a weak, reversible interaction between the Lewis acidic boron and Lewis basic catechol, but without formation of a boronate ester. On adding a tertiary amine base such as $N$-methylmorpholine, a new signal was observed in the ${ }^{19} \mathrm{~F}$ NMR spectrum at $\delta=-126.8 \mathrm{ppm}$, which was assigned to the boronate ester $\mathbf{1 A}$, stabilized by coordination of the nitrogen base at boron. In-line with our previous studies on nanoparticles, $(18 b)$ the concentration of boronate ester was observed to increase with increasing concentration of base (Figure 1c, half-filled circles), reaching a maximum at around $100 \mathrm{mM} \mathrm{N}$-methylmorpholine (corresponding to ca. 20 molar equivalents with respect to boronic acid and catechol).

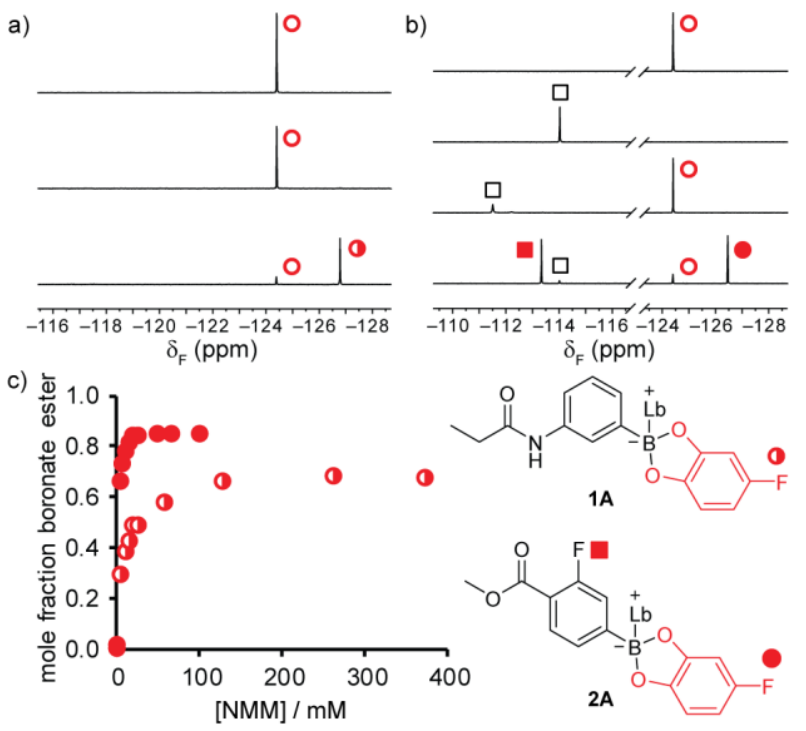


Figure 1. Characterization of boronate ester formation with catechol $\mathbf{A}$ in response to addition of $N$-methylmorpholine (NMM) by ${ }^{19} \mathrm{~F}\left\{{ }^{1} \mathrm{H}\right\}$ NMR $\left(\mathrm{CD}_{3} \mathrm{OD}, 470.1 \mathrm{MHz}, 298\right.$ K). (a) Spectra (top to bottom) of: $\mathbf{A}+500$ mM NMM; $\mathbf{A}+\mathbf{1}$ (no NMM); $\mathbf{A}+\mathbf{1}+500$ mM NMM. (b) Spectra (top to bottom) of: A + 500 mM NMM; 2 + 500 mM NMM; A + 2 (no NMM); A + 2 + 500 mM NMM. Open symbols: uncomplexed components; filled/patterned symbols: boronate ester species. (c) Variation of mole fraction of boronate ester with increasing concentration of NMM for $\mathbf{1 A}$ (half-filled circles) and $\mathbf{2 A}$ (filled circles). Initial concentrations: $[\mathbf{A}]_{0} \approx[\mathbf{1}]_{0}$ or $[\mathbf{2}]_{0} \approx 5 \mathrm{mM}$. See Figures S1 and S4 for NMR spectra showing a wider chemical shift range for $[\mathrm{NMM}]=5,50,500 \mathrm{mM}$; see Figures S13a and S14a for full NMM titration data.

The requirement to introduce such high concentrations of base in order to maximise boronate ester formation is generally undesirable: the rate of aerobic catechol oxidation to quinones (vide supra) is increased under such conditions, and other unforeseen side reactions or interactions may arise when working with more complex systems. Boronic acid $\mathbf{2}$ was therefore prepared to investigate whether electronwithdrawing substituents on the aromatic ring could increase the stabilities of the corresponding boronate esters, thus lowering the concentration of Lewis base required in order to achieve maximum ester formation. The fluorine substituent also provides a spectroscopic probe for boronate ester formation, without requiring fluorine-labelled coupling partners. Titrating each boronic acid with $N$-methylmorpholine and fitting the observed chemical shift changes to a 1:1 binding isotherm gave an estimate of the equilibrium constant for the acid-base interaction $\left(K_{\mathrm{a}}^{\mathrm{NMM}}\right.$, Figure S8), confirming the significantly greater Lewis acidity of $\mathbf{2}\left(K_{\mathrm{a}}^{\mathrm{NMM}}=130 \mathrm{M}^{-1}\right)$ compared to $\mathbf{1}\left(K_{\mathrm{a}}{ }^{\mathrm{NMM}}=36\right.$ $\mathrm{M}^{-1}$ ). Gratifyingly, on titrating $N$-methylmorpholine into a 1:1 solution of 2 and catechol A (ca. $5 \mathrm{mM}$ ), formation of boronate ester $\mathbf{2 A}$ approached a maximum value after only around $20 \mathrm{mM}$ base (ca. 4 molar equivalents with respect to boronic acid and 
catechol, Figure 1c, filled circles), a significant reduction on the value of $100 \mathrm{mM}$ required to reach maximal formation of $\mathbf{1 A}$.

Turning to alternative, non-catechol, coupling partners, we first investigated the response of $\mathbf{C}$ and $\mathbf{D}$ alone to increasing concentrations of $N$-methylmorpholine. The fluorine label in each of $\mathbf{A}, \mathbf{C}$ and $\mathbf{D}$ gives rise to a single ${ }^{19} \mathrm{~F}$ NMR signal. Whereas no change in chemical shift value was observed on introducing up to $500 \mathrm{mM} \mathrm{N}$ methylmorpholine to a solution of $\mathbf{A}\left(5 \mathrm{mM}, \mathrm{CD}_{3} \mathrm{OD}\right.$, Figure $\left.\mathrm{S} 10\right)$, the resonances for both $\mathbf{C}$ and $\mathbf{D}$ were shifted upfield on introducing base (Figure $2 \mathrm{a}, \mathrm{b}$ ). The significantly steeper response to base concentration observed for $\mathbf{C}$ reflects the acidity of the carboxylic acid compared to the most acidic site in $\mathbf{D}$ (presumably the phenolic proton). Fitting to 1:1 binding isotherms gave estimates of equilibrium constants for the deprotonation of each compound by $N$-methylmorpholine ( $K_{\mathrm{a}}{ }^{\mathrm{NMM}}$, Figure S11).(20) It should be noted that the chemical shift change for $\mathbf{C}$ continues to rise marginally at high base concentrations, perhaps indicative of a second deprotonation equilibrium involving the phenolic proton. 

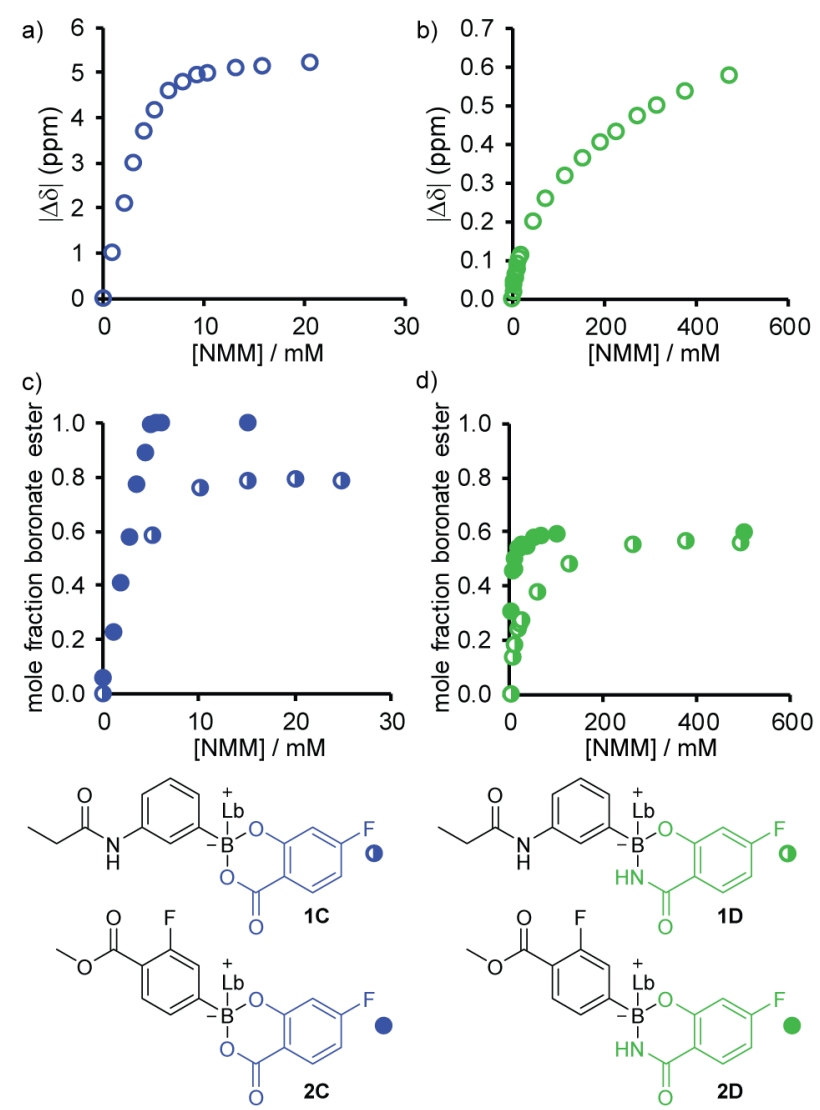

Figure 2. Response of salicylic acid (C) and salicylamide (D) coupling partners to concentration of Lewis base. $(a, b)$ Chemical shift change as a function of concentration of $N$-methylmorpholine (NMM) for (a) C and (b) D. (c) Variation of mole fraction of boronate ester with increasing concentration of NMM for $\mathbf{1 C}$ (half-filled blue circles) and 2C (filled blue circles). (d) Variation of mole fraction of boronate ester with increasing concentration of NMM for 1D (half-filled green circles) and 2D (filled green circles). Initial concentrations of all boronic acids and coupling partners ca. $5 \mathrm{mM}$. See Figures S2, S3, S6, S7 for representative NMR spectra, and Figures S13, S14 for full NMM titration data.

The increased acidities of $\mathbf{C}$ and $\mathbf{D}$ compared to catechol $\mathbf{A}$ are reflected in the formation of boronate complexes with these coupling partners. In the absence of base, a noticeable downfield shift $(\Delta \delta=0.18 \mathrm{ppm})$ was observed for the ${ }^{19} \mathrm{~F}$ resonance of $\mathbf{C}$ on introducing boronic acid $\mathbf{1}$ at a 1:1 stoichiometric ratio, again indicative of a weak association in fast exchange. Titrating this mixture with $N$-methylmorpholine gave rise 
to a new resonance for boronate ester $\mathbf{1 C}$, once more in slow exchange on the NMR timescale with the individual components (Figure S2). In contrast to $\mathbf{1 A}$, the concentration of boronate ester reached a maximum value after adding only $15 \mathrm{mM} \mathrm{N}$ methylmorpholine (ca. 3 molar equivalents, Figure 2c, half-filled circles). Significantly, combining coupling partner $\mathbf{C}$ with the more acidic boronic acid $\mathbf{2}$, a small amount of boronate ester was observed even in the absence of $N$-methylmorpholine (Figure S6), and the saturation concentration of boronate ester $\mathbf{2 C}$ was reached after only 1 molar equivalent of base was added ( $\approx 5 \mathrm{mM}$, Figure $2 \mathrm{c}$, filled circles). Requiring only a stoichiometric amount of base to achieve maximum ester formation - with a coupling partner that does not undergo oxidative side reactions - represents a significant advantage over the original system $\mathbf{1 A}$, which required greater than 20 molar equivalents Lewis base $(\approx 100 \mathrm{mM})$ under the same conditions.

Salicylamide D exhibited a significantly lower tendency to form boronate esters, requiring $>300 \mathrm{mM}($ (1D) and $>50 \mathrm{mM}$ (2D) base to approach maximum ester concentrations, respectively (Figure 2d). When combining $\mathbf{D}$ with $\mathbf{1}$ or $\mathbf{2}$, a low-intensity signal in slow exchange with the isolated components was observed in the absence of base (Figure S3 and S7). However, on introducing increasing quantities of $N$ methylmorpholine, this signal decreased in intensity, concomitant with the growth of a second new signal, also in slow exchange with the isolated components. Eventually, only the second signal, which could be assigned to the expected boronate complex (1D / 2D), was observed. We were unable to unambiguously identify the structure of the initially formed complex, and so considered the combined concentration of both species as reflecting total boronate complex formed at each stage (see Supporting Information). Although the lower acidity of $\mathbf{D}$ compared to $\mathbf{C}$ might account for the different behaviours exhibited by these two salicylate derivatives, comparison with catechol $\mathbf{A}$ 
clearly reveals that acidity alone does not explain the difference in boronate ester stability between these structurally dissimilar coupling partners. Despite being considerably less acidic than $\mathbf{D}$, catechol A requires similar concentrations of $\mathrm{N}$ methylmorpholine to reach maximum ester formation, and furthermore, the concentrations of esters observed suggest that $\mathbf{1 A}$ and $\mathbf{2 A}$ are significantly more stable than their salicylamide counterparts 1D and 2D.

To provide a quantitative comparison of stabilities across a representative range of conditions, apparent formation constants $\left(K_{\mathrm{app}}\right)$ were calculated from the equilibrium concentrations of all species as measured by NMR spectroscopy for each boronate ester at $0,5,50$, and $500 \mathrm{mM} \mathrm{N}$-methylmorpholine in methanol (Figure 3 and Table S1). In agreement with our initial observations, the more electron-deficient boronic acid $\mathbf{2}$ tends to form more stable boronate ester complexes than $\mathbf{1}$ with all partners. For the less acidic coupling partners $\mathbf{A}, \mathbf{B}$ and $\mathbf{D}$, the highest boronate ester stabilities are observed at the highest concentration of base employed (500 mM). Under these conditions the formation constants for catechol derivatives $\left(K_{\text {app }}(\mathbf{1 A})=4000 \mathrm{M}^{-1} ; K_{\text {app }}(\mathbf{2 A})=9700 \mathrm{M}^{-}\right.$ ${ }^{1} ; K_{\text {app }}(\mathbf{2 B})=46000 \mathrm{M}^{-1}$ ) are significantly higher - by approximately an order of magnitude - than their salicylamide counterparts $\left(K_{\text {app }}(\mathbf{1 D})=470 \mathrm{M}^{-1} ; K_{\text {app }}(\mathbf{2 D})=690\right.$ $\left.\mathrm{M}^{-1}\right) .(21)$ This trend is also maintained for the lower stabilities observed at lower concentrations of base, and can be ascribed to the different structures of boronate ester complexes formed by $\mathbf{A}, \mathbf{B}$ and $\mathbf{D}$. Whereas formation of 6-membered cyclic complexes from $\mathbf{D}$ involves an entropic penalty, catechols are pre-organized for formation of cyclic structures, with minimal entropy loss.(16b) Perhaps equally significant, in both cases, condensation with a boronic acid requires disrupting intramolecular hydrogen bonding and intermolecular solvent interactions, which would be expected to be more significant 
for the primary amide and acidic phenol(ate) functionalities in $\mathbf{D}$, compared to the poorly acidic catechol functionality of A.(22)
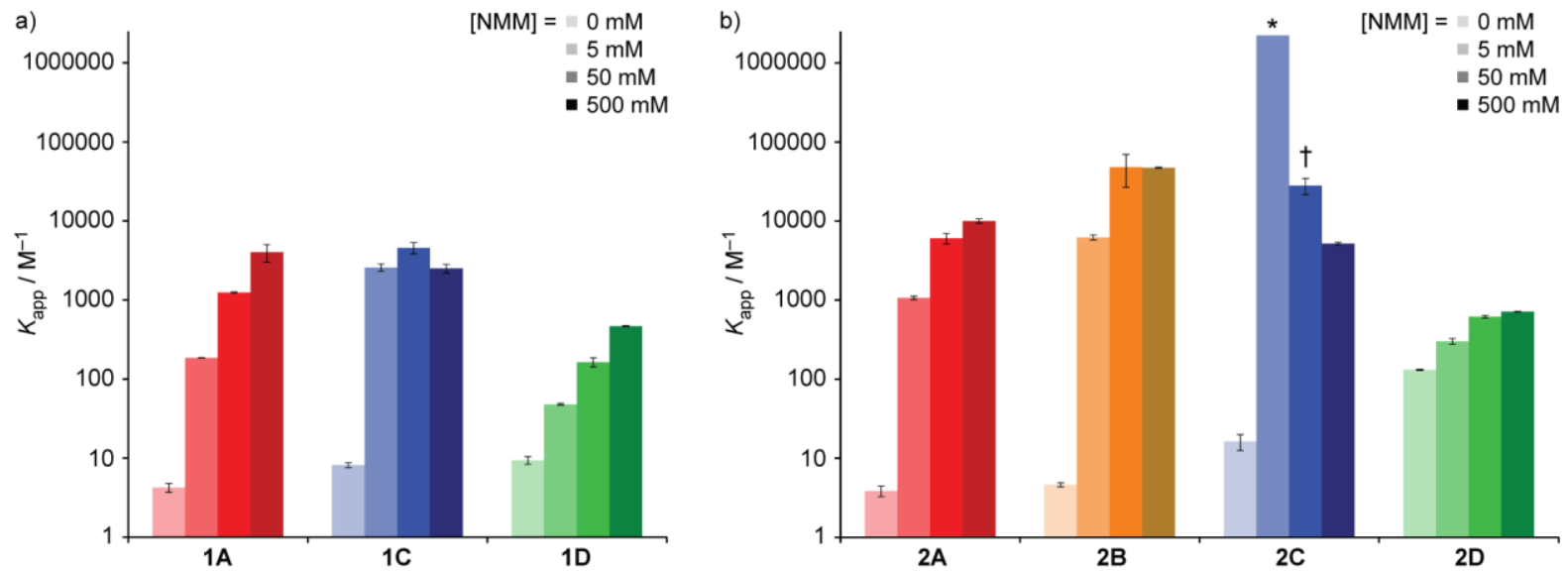

Figure 3. Apparent formation constants $\left(K_{\mathrm{app}}\right)$ for boronate ester complexes $\mathbf{1 A}, \mathbf{1 C}$, 1D, 2A, 2B, 2C, 2D, measured in $\mathrm{CD}_{3} \mathrm{OD}$ with varying concentration of $N$ methylmorpholine (NMM) and initial concentrations of all boronic acids and coupling partners ca. $5 \mathrm{mM}$. Values expressed as mean \pm 1 standard deviation of three independent replicates. For corresponding NMR spectra, see Figures S1-S7. (*) Estimated minimum value for $\mathbf{2 C}$ at $5 \mathrm{mM} \mathrm{NMM}\left(K_{\text {app }}>2.2 \times 10^{6} \mathrm{M}^{-1}\right)$. (†) Value for 2C at $50 \mathrm{mM}$ NMM determined by a competition experiment. See Supporting Information for details.

The influence of Lewis base concentration and boronic acid acidity on boronate complex stabilities can be understood by reference to the minimal scheme of coupled equilibria (Scheme 2a) that is commonly used to describe the interaction between a generic boronic acid (Ba) and diol coupling partner $(\mathbf{P}) \cdot(7-8,12,16 a)$ Coordination of a Lewis base changes the hybridisation at boron from $\mathrm{sp}^{2}$ to $\mathrm{sp}^{3}$. Particularly for complexes with 1,2-diols - which form a 5-membered chelate - this results in a significant relief of ring strain in the ester species, such that these are considerably more acidic than the corresponding boronic acids $K_{\mathrm{a}}(\mathbf{B a P}) \gg>K_{\mathrm{a}}(\mathbf{B a})$, and hence $K\left(\mathbf{B a}^{-} \mathbf{P}\right) \gg>$ $K\left(\mathbf{B a}^{\mathbf{0}} \mathbf{P}\right)$. The overall result is that the proportion of ester observed (i.e. total 
concentration of complexed species, as described by the measured apparent formation constant $\left.K_{\text {app }}\right)$ increases on increasing base concentration, and the dominant ester species is the tetrahedral form $\mathbf{B a}^{-} \mathbf{P}$. Electron poor phenylboronic acids are better able to stabilize the resulting negative charge on boron in the tetrahedral ester complex, but also stabilize the uncomplexed tetrahedral form $\mathrm{Ba}^{-}$, so that the effect of boronic acid $\mathrm{p} K_{\mathrm{a}}$ on the observed ester formation equilibrium depends on both partners in question, as well as the concentration of base.(23) At lower concentrations of Lewis base, the stabilizing effect of increasing acidity at boron is more significant. This is reflected in the relative values of the formation constants for complexes of $\mathbf{2}$ compared to $\mathbf{1}$ : $K_{\text {app }}(\mathbf{2 A}) / K_{\text {app }}(\mathbf{1 A})=5.6$ at low base concentration $([\mathrm{NMM}]=5 \mathrm{mM})$, but only 2.4 at high base concentration $([\mathrm{NMM}]=500 \mathrm{mM}) ; K_{\text {app }}(\mathbf{2 D}) / K_{\text {app }}(\mathbf{1 D})=6.0$ at $[\mathrm{NMM}]=5$ $\mathrm{mM}$, but only 1.5 at $[\mathrm{NMM}]=500 \mathrm{mM}$. a) $R$<smiles>[Y]=[Te]</smiles>

$\mathrm{Ba}^{0}$ $K_{\mathrm{a}}(\mathbf{B a}) \| \pm \mathrm{Lb}$

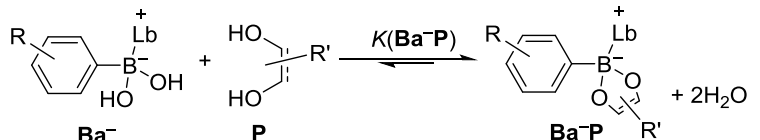

$\mathrm{Ba}^{0} \mathrm{P}$

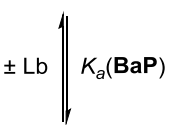

$\mathrm{Ba}^{-} \mathbf{P}$ b)

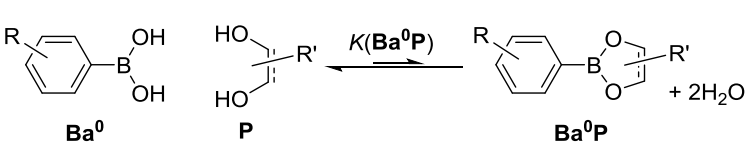

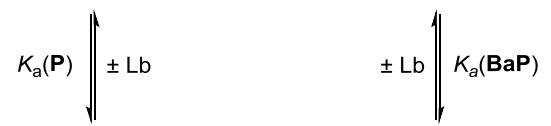

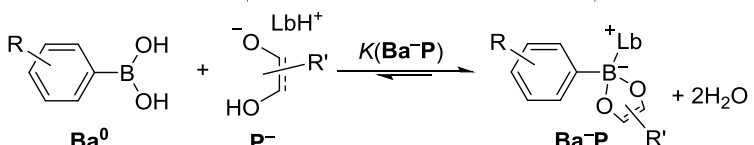

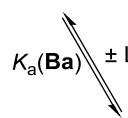

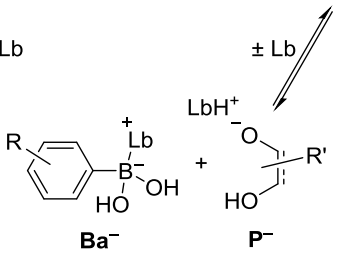

Scheme 2. (a) Simplified general scheme of coupled equilibria describing the interactions between a generic boronic acid (Ba) and a 'non-acidic' coupling partner $\mathbf{P}$ (i.e. $K_{\mathrm{a}}(\mathbf{P})<<K_{\mathrm{a}}(\mathbf{B a})$ ), in the presence of a generic Lewis base (Lb). (b) Expanded scheme of coupled equilibria describing the interactions between a generic boronic acid (Ba) and an 'acidic' coupling partner $\mathbf{P}$ (i.e. $K_{\mathrm{a}}(\mathbf{P})>>K_{\mathrm{a}}(\mathbf{B a})$ ), in the presence of a generic Lewis base (Lb). As the acid-base equilibria are all in fast exchange on the NMR timescale, apparent association constants observed experimentally correspond to 
the total concentrations of complexed and uncomplexed species: $K_{\text {app }}=[\mathbf{B a P}]_{\text {total }} /$ $\left([\mathbf{B a}]_{\text {total }}[\mathbf{P}]_{\text {total }}\right)$.

Salicylic acid coupling partner $\mathbf{C}$ exhibits quite different behaviour. As already noted, maximum formation of boronate ester $\mathbf{1 C}$ is achieved around $15 \mathrm{mM} \mathrm{N}$ methylmorpholine, which is reflected in the single point formation constants measured at base concentrations of $5 \mathrm{mM}\left(K_{\text {app }}(\mathbf{1 C})=2600 \mathrm{M}^{-1}\right)$ and $50 \mathrm{mM}\left(K_{\text {app }}(\mathbf{1 C})=4500 \mathrm{M}^{-}\right.$ $\left.{ }^{1}\right)$. Interestingly, these values do not differ greatly from the maximum equilibrium constant observed for $\mathbf{1 A}$, formed from the highly pre-organized catechol partner $\left(K_{\text {app }}(\mathbf{1 A})=4000 \mathrm{M}^{-1}\right.$, at $\left.[\mathrm{NMM}]=500 \mathrm{mM}\right)$. Even more intriguing, was the observation of a small but significant decrease in stability of complex $\mathbf{1 C}$ at very high Lewis base concentrations $\left(K_{\text {app }}(\mathbf{1 C})=2500 \mathrm{M}^{-1}\right.$, at $\left.[\mathrm{NMM}]=500 \mathrm{mM}\right)$. In combination with boronic acid $\mathbf{2}$, as suggested by our preliminary observations, a small, but appreciable, formation constant can be calculated in the absence of base $\left(K_{\mathrm{app}}(\mathbf{2 C})=\right.$ $16 \mathrm{M}^{-1}$ ). Meanwhile on adding only $5 \mathrm{mM} N$-methylmorpholine, formation of complex 2C is almost entirely quantitative. Neither diluting the system 10 -fold, while maintaining a stoichiometric quantity of base $\left([2]_{0} \approx[\mathbf{C}]_{0} \approx[\mathrm{NMM}]=0.5 \mathrm{mM}\right)$, nor performing a competition experiment in the presence of catechol $\mathbf{A}$ was able to disrupt complex 2C sufficiently to allow measurable concentrations of uncomplexed species, so that only a lower bound for the value of $K_{\text {app }}(\mathbf{2 C})$ can be estimated by NMR analysis ( $>$ $2.2 \times 10^{6} \mathrm{M}^{-1}$, at $[\mathrm{NMM}]=5 \mathrm{mM}$; see Supporting Information Section 2.1 for further details). As observed for $\mathbf{1 C}$, on further increasing the concentration of Lewis base, the stability of boronate ester $\mathbf{2 C}$ decreased, this time quite significantly, so that the formation constant at $500 \mathrm{mM}$ is at least two orders of magnitude less than the maximum value $\left(K_{\text {app }}(\mathbf{2 C})=5000 \mathrm{M}^{-1}\right.$ at $\left.[\mathrm{NMM}]=500 \mathrm{mM}\right)$. 
The observation that high concentrations of base shift the equilibria for boronate complex formation with salicylates back towards the dissociated components can be explained by a more detailed consideration of the thermodynamic scheme and relative acidities of both interacting species.(7, 12c, 12d, 24) Coupling partners A (no measurable interaction), $\mathbf{B}\left(K_{\mathrm{a}}^{\mathrm{NMM}}=9.8 \mathrm{M}^{-1}\right)$ and $\mathbf{D}\left(K_{\mathrm{a}}^{\mathrm{NMM}}=8.9 \mathrm{M}^{-1}\right)$ can be considered to be less acidic than boronic acids $\mathbf{1}\left(K_{\mathrm{a}}^{\mathrm{NMM}}=36 \mathrm{M}^{-1}\right)$ or $\mathbf{2}\left(K_{\mathrm{a}}^{\mathrm{NMM}}=130\right.$ $\mathrm{M}^{-1}$ ), so that the thermodynamic system is well described by Scheme 2a: the most favourable interaction for the Lewis base is at boron; the next most acidic site being the phenol on A, B or D. On the other hand carboxylic acid $\mathbf{C}\left(K_{\mathrm{a}}^{\mathrm{NMM}}=2900 \mathrm{M}^{-1}\right)$ is significantly more acidic than either of the boronic acids. In this system, the first deprotonation involves the coupling partner itself; the second most acidic site being at boron (Scheme 2b). Whichever scheme is applicable, boronate ester formation is most favourable when there is no change in overall charge between the dissociated components and complexed species.(24) Under the conditions employed here, which strongly favour negatively charged tetrahedral boron complexes, formation should be maximized midway between the first and second deprotonations of the isolated components.(24) Consequently, ester formation increases with the concentration of Lewis base initially, but as the $\mathrm{p} K_{\mathrm{a}}$ for the second most acidic site on the isolated components is approached, boronate complexes are destabilized and the equilibria shift to favour higher concentrations of the dissociated species. For $\mathbf{A}, \mathbf{B}$ and $\mathbf{D}$ this regime is not encountered within the range of base concentrations employed here. For highly acidic $\mathbf{C}$, however, complex destabilization occurs at relatively low concentrations of Lewis base. This accounts for the observation that (a) boronate complexes with salicylic acid $\mathbf{C}$ are most stable at intermediate concentrations of base; and (b) the optimum base 
concentration is lower for complex $\mathbf{2} \mathbf{C}$ - formed with the more acidic boronic acid compared to complex 1C.(25)

The dissimilar absolute stabilities of salicylate and catechol boronate complexes, and their distinctive responses to varying concentration of Lewis base, led us to envisage a responsive system where the preferred coupling partner for a boronic acid could be reversibly switched by a simple chemical stimulus. In-line with the measured formation constants (Figure 3, Table S1), combining boronic acid 2, with equimolar quantities of 4-fluorocatechol $\mathbf{A}$, 4-fluorosalicylic acid $\mathbf{C}$, and $N$-methylmorpholine, gave a product mixture consisting almost exclusively of complex 2C (2A:2C 6:94). On increasing the concentration of $\mathrm{N}$-methylmorpholine, the system responded to produce complex $\mathbf{2 A}$ at the expense of $\mathbf{2 C}$. As expected from the respective formation constants, the equilibrium composition could be switched to favour complex $\mathbf{2 A}$ at a ratio of ca. 2:1 (2A:2C) in the presence of $920 \mathrm{mM} N$-methylmorpholine $(\approx 190$ equivalents, Figure S16a, Table S2).

An improved response - in terms of both switching fidelity and reduced concentration of the Lewis base stimulus required - was achieved by changing to the stronger Lewis base $N, N$-diisopropylethylamine (DIPEA).(26) Boronate ester stabilities were found to be more sensitive to changes in Lewis base concentration using the stronger Lewis base (compare Figures S14, S15). Formation of ester 2A was maximised in the presence of ca. 1.8 molar equivalents $N, N$-diisopropylethylamine (Figure S15a, compared to ca. 4 molar equivalents of NMM). With acidic coupling partner $\mathbf{C}$, virtually quantitative formation of boronate ester $\mathbf{2 C}$ was again observed with only 1 molar equivalent $N, N$-diisopropylethylamine (all components ca. $5 \mathrm{mM}$ ), but significant destabilization of complex $\mathbf{2 C}$ was observed even in the presence of small excesses of the Lewis base (Figure S15b). 
Using the stronger Lewis base in the presence of both coupling partners, a binary switching response could be achieved (Figure 4 and Table S4) between equilibrium compositions favouring almost exclusively salicylate complex $\mathbf{2 C}(<5: 95$ $\mathbf{2 A}: \mathbf{2 C}$ at $[\mathrm{DIPEA}]=4.8 \mathrm{mM} \approx 1.0$ molar equivalents $)$ or catechol complex $\mathbf{2 A}(>98: 2$ 2A:2C at [DIPEA] $>90 \mathrm{mM} \approx 19$ molar equivalents).(27) Furthermore, simply neutralizing all but 1 equivalent of the base by addition of $\mathrm{HCl}$ returned the system to favour complex $\mathbf{2 C}$. The process was entirely reversible; by sequential addition of base then acid, the cycle could be repeated at least three times with no significant loss of fidelity (Figure 4 and Table S4).
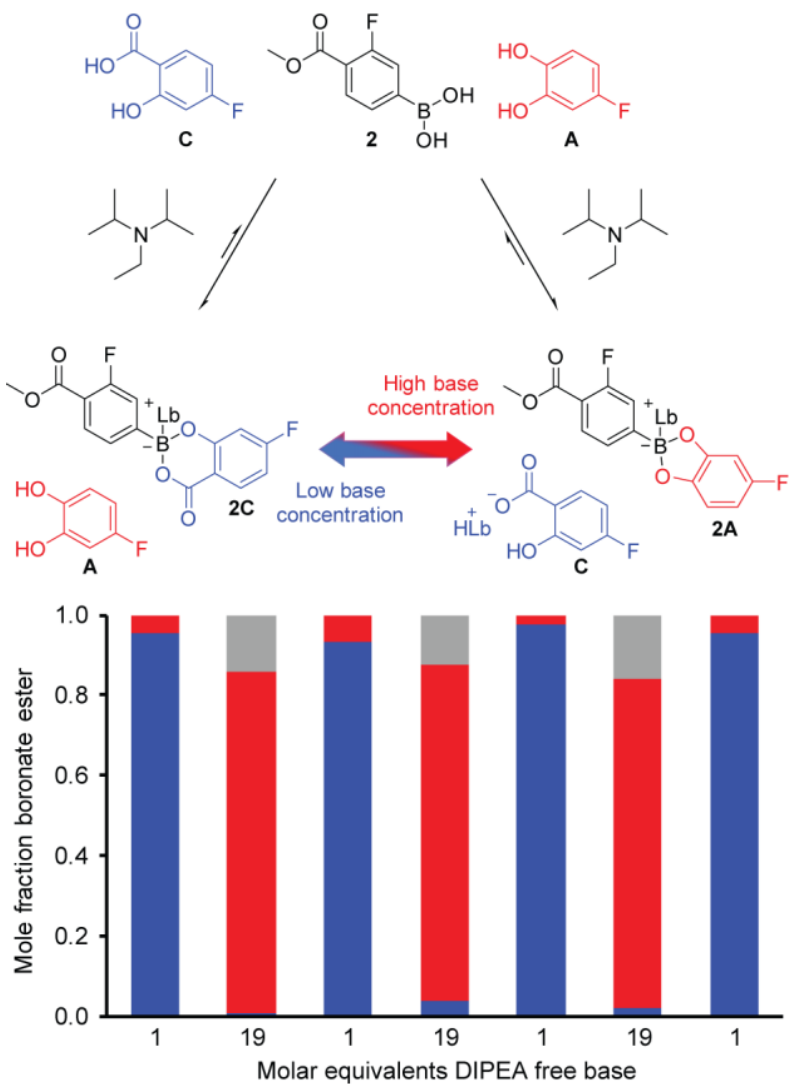

Figure 4. Base-triggered switching of boronate ester selectivity. High fidelity, reversible switching between salicylate boronate complex 2C (blue, low base concentration) and catechol boronate ester $\mathbf{2 A}$ (red, high base concentration) on cyclic variation of the concentration of $N, N$-diisopropylethylamine (DIPEA) free base by alternate addition of DIPEA then $\mathrm{HCl}$. Grey bars: mole fraction of uncomplexed 
boronic acid. Initial concentrations: $[2]_{0}=4.75 \mathrm{mM} \approx[\mathbf{A}]_{0} \approx[\mathbf{C}]_{0}$; molar equivalent values of free base are estimated volumetrically as protonated and free base forms are in fast exchange on the NMR timescale. Concentrations of each species are reported in Table S4.

\section{Conclusions}

Salicylic acids and salicylamides extend the suite of reversible covalent coupling partners for boronic acids in nonaqueous solvents. Compared to commonly exploited 1,2-diphenols, these non-diol motifs are oxidatively stable, and synthetically amenable to derivatization. The corresponding boronate ester complexes span a wide range of stabilities - tuneable according to the concentration and nature of Lewis base present and exhibit maximum formation constants that can be orders of magnitude higher than those achievable with either aromatic or aliphatic diols.

The factors affecting reversible covalent reactions of boronic acids in nonaqueous solvents follow similar principles to the same processes in aqueous solution. Although not previously observed in nonaqueous systems, there is an optimum base concentration for maximising boronate ester stability, which becomes practically relevant for strongly acidic coupling partners such as salicylic acids. Boronate complexes with less acidic coupling partners, such as salicylamides, exhibit weaker overall formation constants, but maintain their maximum stabilities across a wide range of base concentrations. Boronate ester stability can also be manipulated by changing the electronic character of the boronic acid and, as is the case in water, the effect on the observed ester formation constant is a complex function of the components involved and reaction conditions.

Understanding all these effects is crucial to the application of boronate ester dynamic covalent reactivity to create sophisticated functional systems. As a simple 
example, we have achieved repetitive high fidelity reversible switching between two boronate ester complexes formed from one boronic acid and two coupling partners, triggered only by the concentration of a Lewis base. Being able to rationally exploit such behaviours will ultimately allow boronate ester dynamic covalent reactivity to be extended far beyond the traditional domains of saccharide sensing and triggered-release in aqueous environments, or as a reluctantly reversible linker for the construction of cages and framework structures.

\section{Supporting Information}

Detailed experimental procedures; characterization data for all compounds; apparent formation constants and NMR spectra for their determination; Lewis acid-base association titrations for isolated components; full titration data for complex response to concentration of base; constitutional response to base concentration for boronate ester selectivity experiments. The research data supporting this publication can be accessed at http://dx.doi.org/10.17630/ec27242a-dc7c-4b49-bdc1-fec66c1c0125.

\section{Acknowledgements}

Mass spectrometry was carried out at the EPSRC UK National Mass Spectrometry Facility (NMSF) at Swansea University.

\section{Disclosure statement}

No potential conflict of interest was reported by the authors.

\section{Funding}

This work was supported by the EPSRC under Grants [EP/K016342/1 and EP/J500549/1]; the Leverhulme Trust under Grant [RPG-2015-042]; the ERASMUS+ scheme [mobility award to E.B.]; the Royal Society of Edinburgh and Scottish Government [Personal Fellowship to E.R.K.]; and the University of St Andrews.

\section{References}

(1) (a) Rowan, S.J.; Cantrill, S.J.; Cousins, G.R.L.; Sanders, J.K.M.; Stoddart, J.F. Angew. Chem. Int. Ed. 2002, 41, 898-952. (b) Korich, A.L.; Iovine, P.M. Dalton 
Trans. 2010, 39, 1423-1431. (c) Bull, S.D.; Davidson, M.G.; Van den Elsen, J.M.H.; Fossey, J.S.; Jenkins, A.T.A.; Jiang, Y.-B.; Kubo, Y.; Marken, F.; Sakurai, K.; Zhao, J.; James, T.D. Acc. Chem. Res. 2013, 46, 312-326.

(2) We follow here the commonly accepted nomenclature that differentiates between tricoordinate boronic acids/esters and tetracoordinate boronate acid/ester anions. In protic solvents, these species normally co-exist in rapid equilibrium. For expediency we generally refer only to the major species likely to be present (typically boronic acids and boronate esters). Although not formally esters, we also use this term to refer to all boronate complexes involving two $\mathrm{B}-\mathrm{X}$ bonds $(\mathrm{X}=\mathrm{N}, \mathrm{O})$ formed by elminating two molecules of water from the uncomplexed components.

(3) (a) Fujita, N.; Shinkai, S.; James, T.D. Chem. - Asian J. 2008, 3, 1076-1091. (b) Severin, K. Dalton Trans. 2009, 5254-5264. (c) Nishiyabu, R.; Kubo, Y.; James, T.D.; Fossey, J.S. Chem. Commun. 2011, 47, 1124-1150. (d) Kubo, Y.; Nishiyabu, R.; James, T.D. Chem. Commun. 2015, 51, 2005-2020.

(4) (a) Côté, A.P.; Benin, A.I.; Ockwig, N.W.; O'Keeffe, M.; Matzger, A.J.; Yaghi, O.M. Science 2005, 310, 1166-1170. (b) Tilford, R.W.; Gemmill, W.R.; zur Loye, H.C.; Lavigne, J.J. Chem. Mater. 2006, 18, 5296-5301. (c) El-Kaderi, H.M.; Hunt, J.R.; Mendoza-Cortés, J.L.; Côté, A.P.; Taylor, R.E.; O'Keeffe, M.; Yaghi, O.M. Science 2007, 316, 268-272. (d) Colson, J.W.; Woll, A.R.; Mukherjee, A.; Levendorf, M.P.; Spitler, E.L.; Shields, V.B.; Spencer, M.G.; Park, J.; Dichtel, W.R. Science 2011, 332, 228-231.

(5) (a) Jin, S.; Cheng, Y.; Reid, S.; Li, M.; Wang, B. Med. Res. Rev. 2010, 30, 171257. (b) Nishiyabu, R.; Kubo, Y.; James, T.D.; Fossey, J.S. Chem. Commun. 2011, 47, 1106-1123. (c) Hansen, J.S.; Christensen, J.B.; Petersen, J.F.; HoegJensen, T.; Norrild, J.C. Sens. Actuators, B. 2012, 161, 45-79. (d) Wu, X.; Li, Z.; Chen, X.-X.; Fossey, J.S.; James, T.D.; Jiang, Y.-B. Chem. Soc. Rev. 2013, $42,8032-8048$.

(6) (a) Cambre, J.N.; Sumerlin, B.S. Polymer 2011, 52, 4631-4643. (b) Guan, Y.; Zhang, Y. Chem. Soc. Rev. 2013, 42, 8106-8121. (c) Brooks, W.L.A.; Sumerlin, B.S. Chem. Rev. 2016, 116, 1375-1397. (d) Aznar, E.; Oroval, M.; Pascual, L.; Murguia, J.R.; Martinez-Manez, R.; Sancenon, F. Chem. Rev. 2016, 116, 561718.

(7) Peters, J.A. Coord. Chem. Rev. 2014, 268, 1-22. 
(8) Yan, J.; Springsteen, G.; Deeter, S.; Wang, B.H. Tetrahedron 2004, 60, 1120511209.

(9) Spitler, E.L.; Dichtel, W.R. Nature Chem. 2010, 2, 672-677.

(10) (a) Dowlut, M.; Hall, D.G. J. Am. Chem. Soc. 2006, 128, 4226-4227. (b) Greig, L.M.; Slawin, A.M.Z.; Smith, M.H.; Philp, D. Tetrahedron 2007, 63, 23912403.

(11) (a) Burgemeister, T.; Grobeeinsler, R.; Grotstollen, R.; Mannschreck, A.; Wulff, G. Chem. Ber. Recl. 1981, 114, 3403-3411. (b) Wulff, G. Pure Appl. Chem. 1982, 54, 2093-2102. (c) Wulff, G.; Lauer, M.; Bohnke, H. Angew. Chem., Int. Ed. Engl. 1984, 23, 741-742. (d) Zhu, L.; Shabbir, S.H.; Gray, M.; Lynch, V.M.; Sorey, S.; Anslyn, E.V. J. Am. Chem. Soc. 2006, 128, 1222-1232.

(12) (a) Springsteen, G.; Wang, B.H. Tetrahedron 2002, 58, 5291-5300. (b) Bosch, L.I.; Fyles, T.M.; James, T.D. Tetrahedron 2004, 60, 11175-11190. (c) Martinez-Aguirre, M.A.; Villamil-Ramos, R.; Guerrero-Alvarez, J.A.; Yatsimirsky, A.K. J. Org. Chem. 2013, 78, 4674-4684. (d) Furikado, Y.; Nagahata, T.; Okamoto, T.; Sugaya, T.; Iwatsuki, S.; Inamo, M.; Takagi, H.D.; Odani, A.; Ishihara, K. Chem. - Eur. J. 2014, 20, 13194-13202.

(13) Akgun, B.; Hall, D.G. Angew. Chem. Int. Ed. 2016, 55, 3909-3913.

(14) Meadows, M.K.; Roesner, E.K.; Lynch, V.M.; James, T.D.; Anslyn, E.V. Org. Lett. 2017, 19, 3179-3182.

(15) (a) Stolowitz, M.L.; Ahlem, C.; Hughes, K.A.; Kaiser, R.J.; Kesicki, E.A.; Li, G.S.; Lund, K.P.; Torkelson, S.M.; Wiley, J.P. Bioconjugate Chem. 2001, 12, 229-239. (b) Wiley, J.P.; Hughes, K.A.; Kaiser, R.J.; Kesicki, E.A.; Lund, K.P.; Stolowitz, M.L. Bioconjugate Chem. 2001, 12, 240-250. (c) Moffatt, S.; Wiehle, S.; Cristiano, R.J. Hum. Gene Ther. 2005, 16, 57-67. (d) Roberts, M.C.; Hanson, M.C.; Massey, A.P.; Karren, E.A.; Kiser, P.F. Adv. Mater. 2007, 19, 2503-2507. (e) Jay, J.I.; Shukair, S.; Langheinrich, K.; Hanson, M.C.; Cianci, G.C.; Johnson, T.J.; Clark, M.R.; Hope, T.J.; Kiser, P.F. Adv. Funct. Mater. 2009, 19, 2969-2977. (f) Shin, S.B.Y.; Almeida, R.D.; Gerona-Navarro, G.; Bracken, C.; Jaffrey, S.R. Chem. Biol. 2010, 17, 1171-1176. (g) Jay, J.I.; Langheinrich, K.; Hanson, M.C.; Mahalingam, A.; Kiser, P.F. Soft Matter 2011, 7, 5826-5835. (h) Mahalingam, A.; Jay, J.I.; Langheinrich, K.; Shukair, S.; McRaven, M.D.; Rohan, L.C.; Herold, B.C.; Hope, T.J.; Kiser, P.F. Biomaterials 2011, 32, 8343-8355. (i) Ng, D.Y.W.; Arzt, M.; Wu, Y.Z.; Kuan, 
S.L.; Lamla, M.; Weil, T. Angew. Chem. Int. Ed. 2014, 53, 324-328. (j) Arzt, M.; Seidler, C.; Ng, D.Y.W.; Weil, T. Chem. - Asian J. 2014, 9, 1994-2003.

(16) (a) Lorand, J.P.; Edwards, J.O. J. Org. Chem. 1959, 24, 769-774. (b) Pizer, R.; Babcock, L. Inorg. Chem. 1977, 16, 1677-1681.

(17) (a) Joslyn, M.A.; Branch, G.E.K. J. Am. Chem. Soc. 1935, 57, 1779-1785. (b) Branch, G.E.K.; Joslyn, M.A. J. Am. Chem. Soc. 1935, 57, 2388-2394.

(18) (a) della Sala, F.; Kay, E.R. Angew. Chem. Int. Ed. 2015, 54, 4187-4191. (b) Borsley, S.; Kay, E.R. Chem. Commun. 2016, 52, 9117-9120. (c) Kay, E.R. Chem. - Eur. J. 2016, 22, 10706-10716. (d) Edwards, W.; Marro, N.; Turner, G.; Kay, E.R., Chem. Sci. 2018, DOI: 10.1039/C7SC03666C.

(19) (a) Schäfer, H. Z. Anorg. Allg. Chem. 1942, 250, 82-95. (b) Havel, J.; Havelková, L.; Bartušek, M. Chem. Zvesti 1969, 23, 582-588. (c) Mikesova, M.; Bartušek, M. Chem. Zvesti 1978, 32, 472-477. (d) Tanner, D.W.; Bruice, T.C. J. Am. Chem. Soc. 1967, 89, 6954-6971.

(20) A similar titration of B with $N$-methylmorpholine can be tracked by ${ }^{1} \mathrm{H}$ NMR, Figure S11a.

(21) The larger values of $K_{\text {app }}$ for 4-nitrocatechol boronate ester $\mathbf{2 B}$ compared to $\mathbf{2 A}$ are in-line with the higher acidity of $\mathbf{B}$ compared to $\mathbf{A}$ (Figure S11) and previous observations on the relationship between catechol acidity and boronate ester stability. See (12c, 16b) and: Babcock, L.; Pizer, R. Inorg. Chem. 1980, 19, 5661.

(22) Hunter, C.A. Angew. Chem. Int. Ed. 2004, 43, 5310-5324.

(23) Apparent boronate ester formation constants have been observed to both increase $(12 c, 21)$ or decrease $(12 d, 13)$ on increasing Lewis acidity of the boronic acid. See references (7-8) for further discussion.

(24) In terms of $\mathrm{pH}$, this can be expressed as boronate ester formation is greatest at $\mathrm{pH}=\left(\mathrm{p} K_{\mathrm{a}} 1+\mathrm{p} K_{\mathrm{a}} 2\right) / 2$. See $(7,12 c, 12 d)$ and: Vanduin, M.; Peters, J.A.; Kieboom, A.P.G.; Vanbekkum, H. Tetrahedron 1984, 40, 2901-2911.

(25) Although this effect has recently been highlighted in studies of boronic acid esters formed with acidic diphenols in aqueous solution [see $(7,8,12 b, 12 c)$, the same phenomenon had also previously been observed with non-diol coupling partners for boric acid, which also decompose at $\mathrm{pH}$ values approaching the second most acidic proton in the system: aliphatic $\alpha$-hydroxyacids and dicarboxylic acids [see (24) and: (a) Rebstockova, M.; Bartusek, M. Collect. 
Czech. Chem. Commun. 1977, 42, 627-636], salicylic acid,(19a)

salicylamide, $(19 d)$ as well as acidic diphenols with boric acid: (b) Havelkova, L.; Bartusek, M. Collect. Czech. Chem. Commun. 1968, 33, 4188-4197.

(26) The $\mathrm{p} K_{\mathrm{a}}$ of the $\mathrm{N}, \mathrm{N}$-diisopropylethylammonium conjugate acid is approximately 3 units higher than that for $N$-methylmorpholinium.

(27) In a separate titration experiment, we determined that the proportion of $\mathbf{2 A}$ was maximised at ca. $90 \%$ for $[\mathrm{DIPEA}] \geq 45 \mathrm{mM}$ ( $\approx 9$ equivalents). At this point, no uncomplexed boronic acid was observed. The ratio $\mathbf{2 A}: \mathbf{2 C}$ was further increased at higher base concentrations to $\mathbf{9 8 : 2}$ as a result of a reduction in the amount of 2C observed, concomitant with the appearance of a modest amount of uncomplexed boronic acid (Table S3, Figure S16b). 
Scheme 1. Molecular structures of model electron rich (1) and electron poor (2) phenylboronic acids; catechol (A, B), salicylic acid (C) and salicylamide (D) coupling partners. See Chart S1 for the structures of all boronate ester complexes studied.

Scheme 2. (a) Simplified general scheme of coupled equilibria describing the interactions between a generic boronic acid (Ba) and a 'non-acidic' coupling partner $\mathbf{P}$ (i.e. $K_{\mathrm{a}}(\mathbf{P})<<K_{\mathrm{a}}(\mathbf{B a})$ ), in the presence of a generic Lewis base (Lb). (b) Expanded scheme of coupled equilibria describing the interactions between a generic boronic acid (Ba) and an 'acidic' coupling partner $\mathbf{P}$ (i.e. $K_{\mathrm{a}}(\mathbf{P})>>K_{\mathrm{a}}(\mathbf{B a})$ ), in the presence of a generic Lewis base (Lb). As the acid-base equilibria are all in fast exchange on the NMR timescale, apparent association constants observed experimentally correspond to the total concentrations of complexed and uncomplexed species: $K_{\text {app }}=[\mathbf{B a P}]_{\text {total }} /$ $\left([\mathbf{B a}]_{\text {total }}[\mathbf{P}]_{\text {total }}\right)$.

Figure 1. Characterization of boronate ester formation with catechol $\mathbf{A}$ in response to addition of $N$-methylmorpholine (NMM) by ${ }^{19} \mathrm{~F}\left\{{ }^{1} \mathrm{H}\right\}$ NMR $\left(\mathrm{CD}_{3} \mathrm{OD}, 470.1 \mathrm{MHz}, 298\right.$ K). (a) Spectra (top to bottom) of: $\mathbf{A}+500$ mM NMM; $\mathbf{A}+1$ (no NMM); $\mathbf{A}+\mathbf{1}+500$ mM NMM. (b) Spectra (top to bottom) of: A + 500 mM NMM; $2+500$ mM NMM; A + 2 (no NMM); A + 2 + 500 mM NMM. Open symbols: uncomplexed components; filled/patterned symbols: boronate ester species. (c) Variation of mole fraction of boronate ester with increasing concentration of NMM for $\mathbf{1 A}$ (half-filled circles) and $\mathbf{2 A}$ (filled circles). Initial concentrations: $[\mathbf{A}]_{0} \approx[\mathbf{1}]_{0}$ or $[\mathbf{2}]_{0} \approx 5 \mathrm{mM}$. See Figures $\mathrm{S} 1$ and S4 for NMR spectra showing a wider chemical shift range for $[\mathrm{NMM}]=5,50,500 \mathrm{mM}$; see Figures S13a and S14a for full NMM titration data.

Figure 2. Response of salicylic acid (C) and salicylamide (D) coupling partners to concentration of Lewis base. $(a, b)$ Chemical shift change as a function of concentration of $N$-methylmorpholine (NMM) for (a) C and (b) D. (c) Variation of mole fraction of boronate ester with increasing concentration of NMM for $\mathbf{1 C}$ (half-filled blue circles) and 2C (filled blue circles). (d) Variation of mole fraction of boronate ester with increasing concentration of NMM for 1D (half-filled green circles) and 2D (filled green circles). Initial concentrations of all boronic acids and coupling partners ca. $5 \mathrm{mM}$. See Figures S2, S3, S6, S7 for representative NMR spectra, and Figures S13, S14 for full NMM titration data. 
Figure 3. Apparent formation constants $\left(K_{\mathrm{app}}\right)$ for boronate ester complexes 1A, 1C, 1D, 2A, 2B, 2C, 2D, measured in $\mathrm{CD}_{3} \mathrm{OD}$ with varying concentration of $\mathrm{N}$ methylmorpholine (NMM) and initial concentrations of all boronic acids and coupling partners ca. $5 \mathrm{mM}$. Values expressed as mean \pm 1 standard deviation of three independent replicates. For corresponding NMR spectra, see Figures S1-S7. (*) Estimated minimum value for $2 \mathrm{C}$ at $5 \mathrm{mM} \mathrm{NMM}\left(K_{\text {app }}>2.2 \times 10^{6} \mathrm{M}^{-1}\right) .(\dagger)$ Value for 2C at $50 \mathrm{mM}$ NMM determined by a competition experiment. See Supporting Information for details.

Figure 4. Base-triggered switching of boronate ester selectivity. High fidelity, reversible switching between salicylate boronate complex 2C (blue, low base concentration) and catechol boronate ester $\mathbf{2 A}$ (red, high base concentration) on cyclic variation of the concentration of $N, N$-diisopropylethylamine (DIPEA) free base by alternate addition of DIPEA then $\mathrm{HCl}$. Grey bars: mole fraction of uncomplexed boronic acid. Initial concentrations: $[2]_{0}=4.75 \mathrm{mM} \approx[\mathbf{A}]_{0} \approx[\mathbf{C}]_{0} ;$ molar equivalent values of free base are estimated volumetrically as protonated and free base forms are in fast exchange on the NMR timescale. Concentrations of each species are reported in Table S4. 